\title{
Distribution of Interaural Time Difference in the Barn Owl's Inferior Colliculus in the Low- and High-Frequency Ranges
}

\author{
Hermann Wagner, Ali Asadollahi, Peter Bremen, Frank Endler, Katrin Vonderschen, and Mark von Campenhausen \\ Institute for Biology II, Rheinisch-Westfälische Technische Hochschule Aachen, D-52074 Aachen, Germany
}

Interaural time differences are an important cue for azimuthal sound localization. It is still unclear whether the same neuronal mechanisms underlie the representation in the brain of interaural time difference in different vertebrates and whether these mechanisms are driven by common constraints, such as optimal coding. Current sound localization models may be discriminated by studying the spectral distribution of response peaks in tuning curves that measure the sensitivity to interaural time difference. The sound localization system of the barn owl has been studied intensively, but data that would allow discrimination between currently discussed models are missing from this animal. We have therefore obtained extracellular recordings from the time-sensitive subnuclei of the barn owl's inferior colliculus. Response peaks were broadly scattered over the physiological range of interaural time differences. A change in the representation of the interaural phase differences with frequency was not observed. In some neurons, response peaks fell outside the physiological range of interaural time differences. For a considerable number of neurons, the peak closest to zero interaural time difference was not the behaviorally relevant peak. The data are in best accordance with models suggesting that a place code underlies the representation of interaural time difference. The data from the high-frequency range, but not from the low-frequency range, are consistent with predictions of optimal coding. We speculate that the deviation of the representation of interaural time difference from optimal-coding models in the low-frequency range is attributable to the diminished importance of low frequencies for catching prey in this species.

Key words: sound localization; model; auditory; midbrain; interaural time disparity; sensory map

\section{Introduction}

Interaural time differences (ITDs) are an important cue for azimuthal sound localization (Yin and Kuwada, 1984; Bala et al., 2003; McAlpine, 2005) and are the only stimulus parameter that influences the azimuthal component of head turns in the barn owl (Poganiatz et al., 2001). Neurons that vary their response with ITD are candidates for the functional representation of ITD (Rose et al., 1966; Goldberg and Brown, 1969; Moiseff and Konishi, 1981, 1983; Yin and Kuwada, 1984; Fitzpatrick et al., 1997; Keller and Takahashi, 2005).

Several models explain the mechanisms underlying the representation of ITD. For example, Jeffress (1948) proposed that ITD is extracted from phase-locked monaural signals in different frequency channels by the action of delay lines and binaural coincidence detectors. In the Jeffress model, many neurons, exhibiting response peaks at different ITDs, form a map of ITDs. We refer to this model as "place-code model." Many follow-up models have used these principles (Colburn et al., 1990; Joris et al., 1998;

Received Sept. 27, 2005; revised Feb. 6, 2007; accepted Feb. 27, 2007

This work was sponsored by the Deutsche Forschungsgemeinschaft. Hermann Wagner thanks his mentors at different institutions [Mark Konishi (California Institute of Technology, Pasadena, CA), Kuno Kirschfeld (Max Planck Institute for Biological Cybernetics, Tubingen, Germany), and Geoffrey Manley (Technical University of Munich, Garching, Germany)] for giving him the opportunity to work in their laboratories. We thank Catherine Carr, Benedikt Grothe, and Eli Nelken for their help in various phases of this project.

Correspondence should be addressed to Prof. Dr. Hermann Wagner, Institute for Biology II, Rheinisch-Westfälische Technische Hochschule Aachen, Kopernikusstrasse 16, D-52074 Aachen, Germany. E-mail: wagner@bio2.rwth-aachen.de.

DOI:10.1523/JNEUROSCI.5250-06.2007

Copyright $\odot 2007$ Society for Neuroscience $\quad$ 0270-6474/07/274191-10\$15.00/0
Grau-Serrat et al., 2003; Batra and Yin, 2004). Recently, the importance of the Jeffress model for sound localization was challenged by data suggesting that the slopes rather than the peaks in the ITD curves are important (McAlpine et al., 2001; Brand et al., 2002; Hancock and Delgutte, 2004; Stecker et al., 2005; Zhou et al., 2005; Siveke et al., 2006). This led to the suggestion that only two broad, hemispheric channels that act in a push-pull manner underlie the representation of auditory space. We refer to this later model as "slope-code model." Slope-code models suggest that the range of ITD detectors is tightly restricted, with an absence of neurons tuned to ITDs beyond one-half of a cycle of their frequency. This limit is known as the $\pi$ limit. Thompson et al. (2006) reported evidence consistent with a $\pi$ limit in humans. Harper and McAlpine (2004) applied optimal-detection theory to the problem of ITD representation. They predicted a concentration of the mean interaural phase difference (MIPD) in the low-frequency range, and a map of ITD containing neurons with many homogeneously distributed response peaks in the ITD tuning curves in the high-frequency range.

Most data in the barn owl have been obtained from the highfrequency range $(>2-3 \mathrm{kHz})$, and not enough data are available from the low-frequency range (Wagner et al., 2002; Carr and Koppl, 2004). The aim of this study is to provide more data to allow discrimination between the two specific model types mentioned above. We study the representation of ITD in the inferior colliculus of the barn owl, which contains a map of auditory space (Knudsen and Konishi, 1978a,b). The inferior colliculus (IC) consists of three subnuclei that are sensitive to ITD: the core of 
the central nucleus (ICCc), the lateral shell of the central nucleus (ICCls), and the external nucleus (ICX) (Wagner et al., 2003). The ICCc is especially interesting, because this nucleus contains both low- and high-best frequency neurons, whereas the ICX contains only high-frequency neurons and the ICCls has an intermediate representation.

\section{Materials and Methods}

Data were obtained from 72 adult anesthetized barn owls of either sex over a period of 21 years at four different locations (California Institute of Technology, Pasadena, CA; Max Planck Institute for Biological Cybernetics, Tubingen, Germany; Technical University of Munich, Garching, Germany; Rheinisch-Westfälische Technische Hochschule Aachen, Aachen, Germany). The procedures conformed to National Institutes of Health principles of animal care and were approved by local authorization committees (for details, see descriptions in Wagner et al., 1987, 2002; Wagner and Takahashi, 1990; Bremen et al., 2006).

Standard electrophysiological techniques were used to record extracellularly from multiunits and single units in the IC. Electrodes were advanced from dorsal to ventral through the IC. This direction is almost perpendicular to the frequency layers and parallel to an ITD array (Wagner et al., 1987). In this way, many frequencies could be sampled in a single penetration. Different ITDs were obtained by moving the electrode along the anteroposterior and mediolateral axes. Most of the data from the ICX and the ICCls, and some of the data for ICCc were collected for projects having different scopes (Wagner et al., 1987, 1994, 2002; Takahashi et al., 1989; Wagner, 1990, 1993; Wagner and Takahashi, 1990, 1992; Kautz and Wagner, 1998; Wagner and von Campenhausen, 2002; Bremen et al., 2006). In addition, the ICCc was systematically sampled for this project to specifically obtain neurons tuned to different ITDs and frequencies.

Auditory stimuli were presented dichotically via earphones. Stimuli were either broadband noise (typically $0.1-25 \mathrm{kHz}$ or low-pass filtered at $20 \mathrm{kHz}$ ) or tones. The duration of the stimuli was $100 \mathrm{~ms}$ with an on and off ramp of $5 \mathrm{~ms}$. By varying the ITD in steps that allowed sampling of at least four data points per period at the best frequency (BF) of a unit, the responses as a function of ITD were quantified (ITD curves). For the collection of isointensity frequency response curves, the frequency was varied in steps of $100,200,300$, or $500 \mathrm{~Hz}$, depending on the BF of a unit. The range of frequencies tested typically covered $>1$ octave around the $\mathrm{BF}$ of the unit and often spanned from 200 to $10,000 \mathrm{~Hz}$ (i.e., included most of the barn owl's hearing range). The tuning to interaural level difference (ILD) was measured by typically varying ILDs from $-20 \mathrm{~dB}$ (left louder) to $+20 \mathrm{~dB}$ (right louder) in steps of $4 \mathrm{~dB}$. ILD curves were assembled from these data. The sound level in the recordings was typically between 10 and $30 \mathrm{~dB}$ above the response threshold of a unit. In some units, also rate-level functions, either with monaural or binaural stimulation or both, were recorded.

The ITD range in the barn owl as derived from recording head-related transfer functions ("physiological ITD range") covers about $\pm 250 \mu \mathrm{s}$ (Keller et al., 1998; Poganiatz et al., 2001; von Campenhausen and Wagner, 2006). The tuning to ITD was quantified by the side-peaksuppression ratio, the peak-to-trough ratio, and, in many cases, by circular statistics (Goldberg and Brown, 1969; Yin and Kuwada, 1984; Takahashi and Konishi, 1986; Wagner et al., 1987). In the latter case, the ITD of the stimulus was converted into an interaural phase difference (IPD) by multiplying a given ITD with the frequency of the stimulus tone or, in case of stimulation with noise, the BF of the neuron: IPD (cycles) = ITD (seconds) $\times$ BF (hertz). IPD is a circular measure, and IPD values were used together with the spike numbers to obtain the strength of tuning as expressed by the vector strength and the direction of the tuning as signified by the MIPD. We restricted the tonal stimuli to frequencies that had periods that were multiples of the ITD step. In this way, we always obtained MIPDs that covered full periods. One disadvantage of this method, however, lies in the introduction of a sampling bias by only using specific stimulation frequencies. If the ITD was varied over several full periods, the data over a range that covered multiples of a full period were analyzed. The MIPD was limited to a range from -0.5 to +0.5 cycles. Afterward, the absolute value was calculated because of the spatial situation a symmetrical distribution around 0 cycles of MIPD is expected, if data from both brain sides are taken into account.

At the end of an experiment, the animal was typically perfused, the brain was removed, and anatomical data were obtained that helped with judging of the location of the recordings. In some cases, lesions were made so that the precise location of a recording was known.

\section{Results}

Data from 2051 units from three time-sensitive subnuclei of the inferior colliculus were included in this study. A total of 835 units were from the ICCc, 598 units were localized to the ICCls, and 618 units were from the ICX. Approximately $20 \%$ of the 2051 units were single units. Multiunits and single units could be pooled, because the peaks in the ITD tuning curves obtained with multiunit or single unit isolation occurred at the same ITD (Wagner et al., 1987, 2002).

\section{Basic data}

After a recording site was reached, responses were typically characterized audiovisually by varying ITD, ILD, and frequency, and by stimulating the left and right ears alone. These responses were noted in the protocol notebook. Afterward, several tuning curves were recorded and the responses stored on disk. Typically, first an ITD tuning curve with noise stimulation was obtained (Fig. $1 A$ ). ITD tuning curves were generally cyclic. The tuning curve displayed in Figure $1 \mathrm{~A}$ exhibited two response maxima occurring within the range of ITDs tested, one at $-1500 \mu$ s and the other at $1500 \mu \mathrm{s}$. The period of $3000 \mu$ s indicated a tuning to a BF of 333 $\mathrm{Hz}$. The binaural isointensity frequency response curve, obtained with an ITD of $1500 \mu$ s, indeed revealed a BF slightly higher than $300 \mathrm{~Hz}$ (Fig. 1B). Because the period in the ITD curve typically specified the BF of a unit well, the BF was determined from the period in the ITD curve in those cases in which a frequency tuning curve was not obtained. The BF served as stimulation frequency to obtain a tonal ITD tuning curve (Fig. 1C). The responses shown in Figure 1 were from a unit in ICC. The BF of $333 \mathrm{~Hz}$ suggested that this unit came from the low-frequency end. The ITD and IPD tuning curves (Fig. $1 A, C$ ) exhibited response minima around 0 cycles. The response maxima occurred close to \pm 0.5 cycles (Fig. $1 C$ ).

\section{Physiological characteristics of the responses in the different subnuclei of the IC}

In all three subnuclei, well tuned ITD curves were obtained across the whole frequency range. The physiological differences in the responses of the neurons in the three subnuclei to sound were used for sorting the neurons into the different subnuclei of the IC. Because the sorting criteria have been published in detail previously, only a brief overview will be given here (for additional details, see supplemental material, available at www.jneurosci.org). Only units for which the sorting criteria gave clear results were included in the study.

Neurons in the ICCc were narrowly tuned to frequency (Fig. $1 B)$. When the ITD was varied, the response of the neurons changed in a cyclic manner to stimulation with noise (Fig. 2A). All peaks typically had similar heights. Neurons were arranged in dorsoventral arrays, so that one array represented one ITD, the array-specific ITD (Figs. 2B, 3A). The array-specific ITD corresponded to slope of the regression line in the phase-frequency plots (Figs. $2 B, 3,4 B$ ). The array-specific ITDs in the ICCc represented ipsilateral locations (i.e., arrays recorded on the right brain side had a positive array-specific ITD), whereas arrays re- 

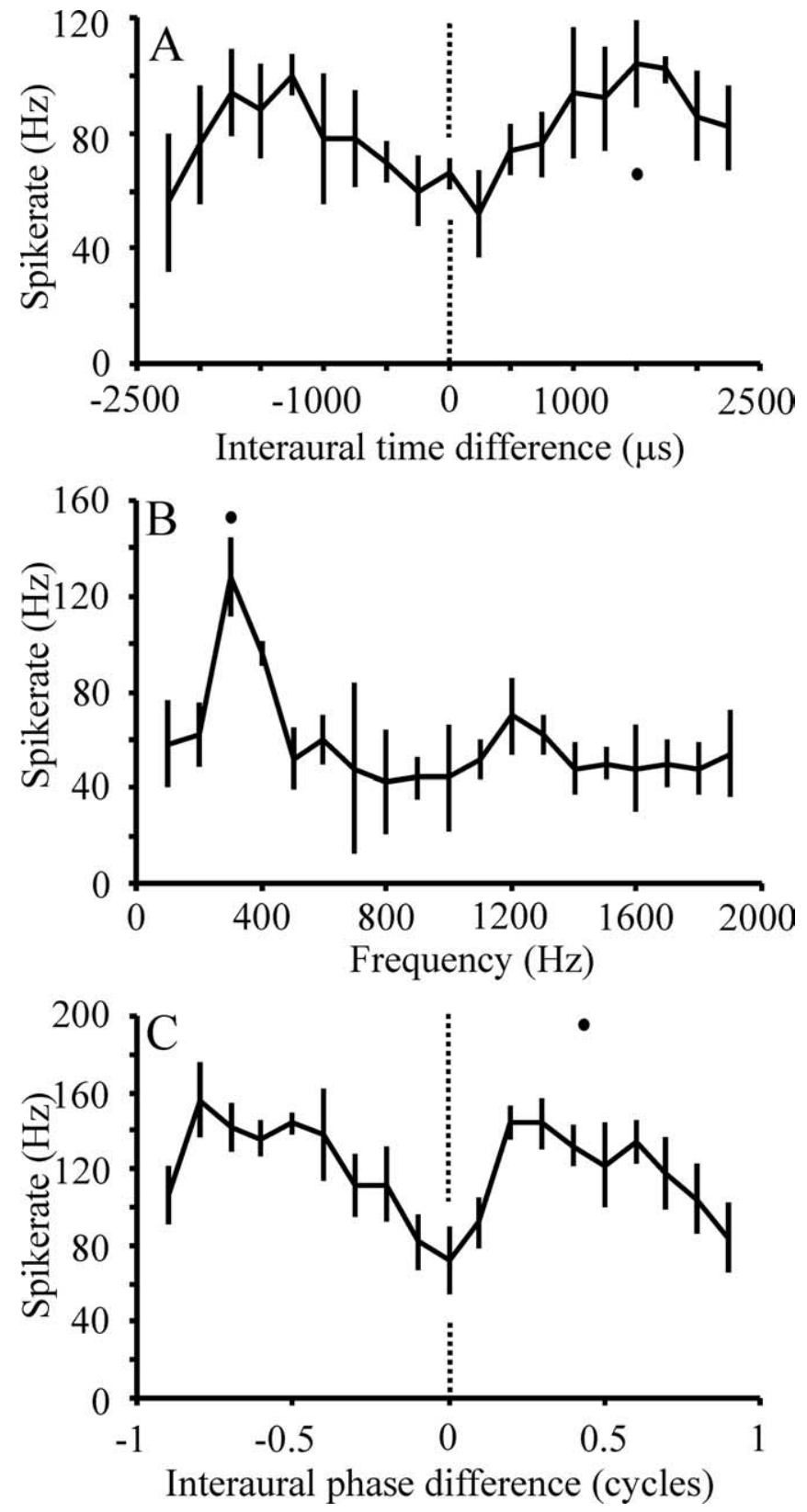

Figure 1. Responses of a low-frequency ICCc neuron. $A$, ITD tuning curve obtained with noise stimulation. $\boldsymbol{B}$, Isointensity frequency response function. The responses were obtained with binaural stimuli at the best ITD in the noise-tuning curve $(1500 \mu \mathrm{s}$; see dot in $\boldsymbol{A})$. The best frequency was at $300 \mathrm{~Hz}$ (see dot in $\boldsymbol{B}$ ). C, IPD curve. The tonal stimulus had a frequency of 333 $\mathrm{Hz}$ and a corresponding period of $3000 \mu \mathrm{s}$. Therefore, an ITD step of $300 \mu \mathrm{s}$ was chosen. To assemble the IPD tuning curves, the ITD was varied from -2700 to $+2700 \mu$ s, and the spikes evoked by a given ITD were counted. The ITD was converted to an IPD by dividing a given ITD by the period ( $3000 \mu \mathrm{s}$ ) belonging to the stimulus tone. Note that the ITD value of the peak in the ITD function (see dot in $\boldsymbol{A}$ ) or the MIPD (0.41) (see dot in $\boldsymbol{C}$ ) and the best or the stimulation frequency ( $300 \mathrm{~Hz}$; see dot in $\boldsymbol{B}$ or $333 \mathrm{~Hz}$ ) constitute one data point for the plots in Figures 8 or 9. Each stimulus was repeated five times.

corded on the left brain side had a negative array-specific ITD (Figs. $2 B, 3 A$ ). In this study, the array-specific ITD was found to be a very reliable criterion, apart from a narrow frontal range (about $\pm 40 \mu \mathrm{s}$ ). Therefore, the array-specific ITD was used as the prime criterion for sorting cells into ICCc and ICCls, respectively, whenever possible.

The responses of the neurons in the next station, the ICCls, were similar to the responses of ICCc neurons in their ITD sen-
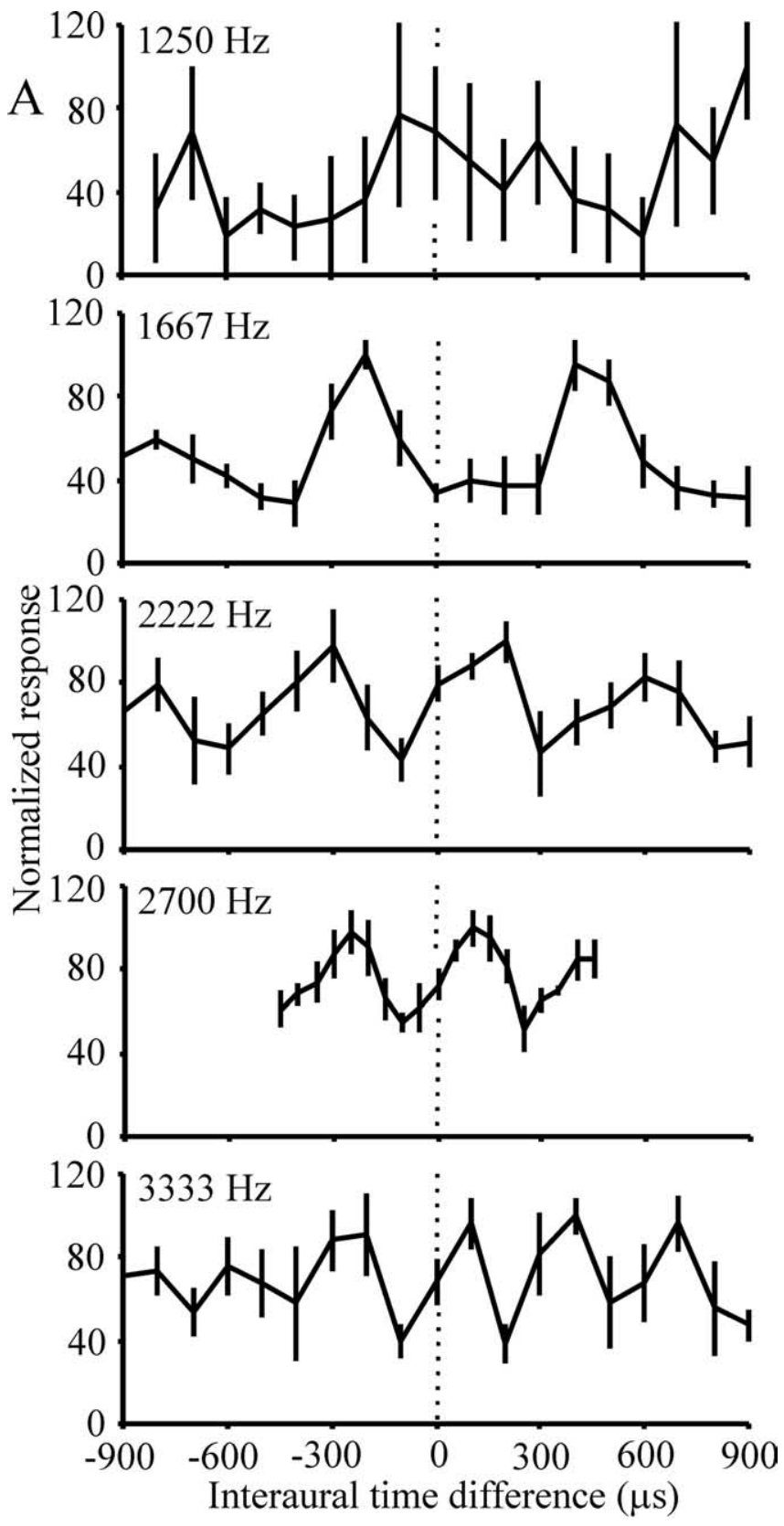

B

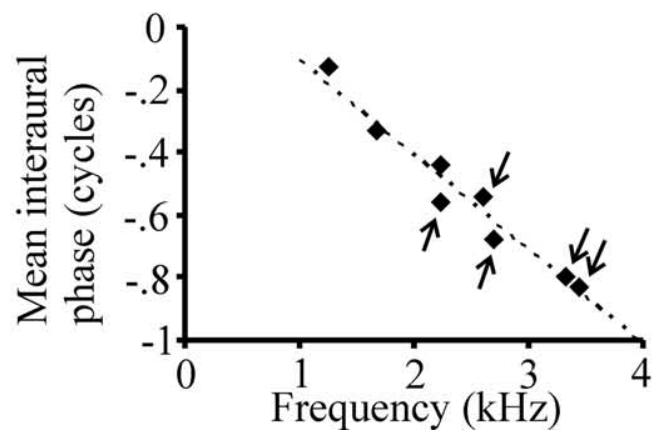

Figure 2. ITD tuning in a dorsoventral penetration through ICCC. $A$, ITD curves from five units tuned to different frequencies (best frequency is noted in insets) from the left brain side. $\boldsymbol{B}$, The phase-frequency plot, including all 8 units recorded in the penetration, indicated a linear relation (correlation coefficient $r=0.98$ ), an array-specific ITD of $-302 \mu$ s, and a characteristic phase of 0.19 . The arrows point to units with peaks outside the $\pi$ limit. 

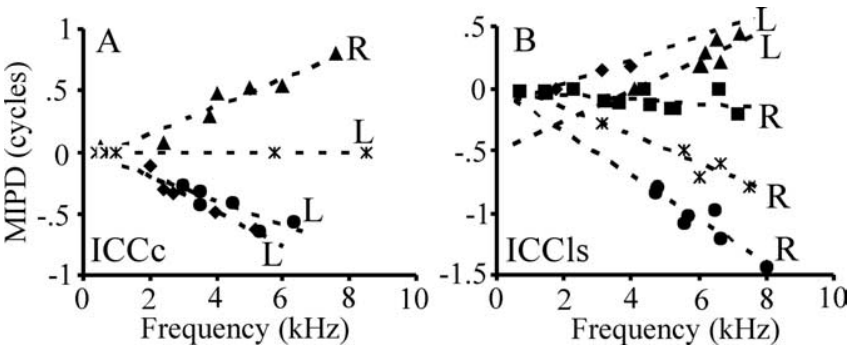

Figure 3. Array-specific ITDs. In ICCC $(\boldsymbol{A})$ and ICCls $(\boldsymbol{B})$, neurons tuned to different frequencies formed functional arrays, so that they all share a common ITD, the array-specific ITD. The values of the array-specific ITD are indicated by the slope of the regression lines, with positive slopes indicating right side leading. Note that the sign of the array-specific ITD indicated ipsilateral locations in ICCC $(\boldsymbol{A})$ and contralateral locations in ICCls $(\boldsymbol{B})$. Data from four penetrations are shown in $\boldsymbol{A}$, whereas in $\boldsymbol{B}$ responses from five penetrations were included. $R$, Recording from the right brain side; $L$, recording from the left brain side. Different penetrations are specified by different symbols.

sitivity and tonotopic organization (compare Figs. 2, 4) but were, in addition, tuned to ILD. Neurons in the ICCls were also arranged in arrays, but the array-specific ITDs in this nucleus represented contralateral locations in space (Figs. $3 B, 4$ ).

The ICCls projects to the ipsilateral ICX. ITD curves obtained with noise stimuli from ICX neurons were characterized by a main peak and side peaks (Fig. 5A). The main peak may also be found by recording ITD curves at many different frequencies and calculating the characteristic delay of a unit. Both methods were used. The tone ITD curve in ICX was cyclic, as were the tuning curves in ICC (Fig. 5C). When lesions were made in the ICX, owls had deficits localizing positions corresponding to the main peak. Thus, the main peak appears to be the behaviorally relevant peak (Wagner, 1993). In the ICX, in contrast to the ICCls, the ILD tuning curves were bell-shaped (Fig. 5B), and the neurons were broadly tuned to frequency (Knudsen, 1984).

\section{Distribution of ITD peaks and best frequencies in ICCc}

In the ICCc, neurons were found with best frequencies ranging from 275 to $8500 \mathrm{~Hz}$, although responses could be obtained from 100 to $9500 \mathrm{~Hz}$. In each frequency band, peaks in the ITD curves occurred at many ITDs. The largest ITD observed was $1500 \mu \mathrm{s}$ (Fig. 1A), well outside the physiological ITD range. We concentrate here on the low-frequency range (neurons with $\mathrm{BFs}<2-3$ $\mathrm{kHz}$ ), because in this range, optimal coding predicted specific, nonuniform distributions (Harper and McAlpine, 2004). How big is the variability in the distribution of ITD peaks with frequency? We tested two extreme cases: First, we asked whether neurons tuned to a narrow range of frequencies exhibit peaks at different ITDs. In our data, sample units having a BF of $400 \mathrm{~Hz}$ exhibited peaks in the ITD curves at $0,-200,-400,-600 \mu \mathrm{s}$, among others (Fig. 6A). The slope and the relative height of the ITD curve at 0 ITD was variable. Likewise, in the $800 \mathrm{~Hz}$ band, peaks in the ITD curves occurred from -625 to $+500 \mu \mathrm{s}$. Responses of three units with peaks at 0,125 , and $300 \mu$ s are shown in Figure $6 B$. Again, many different slopes and relative response heights were observed at 0 ITD. However, our observations indicated a reduction of the range of available ITDs in the $800 \mathrm{~Hz}$ band compared with the $400 \mathrm{~Hz}$ band. A similar trend as for the 400 and $800 \mathrm{~Hz}$ bands was seen for the units having best frequencies between 1600 and $1700 \mathrm{~Hz}$ (Fig. 6C). The relevant peaks were found from -300 to $300 \mu \mathrm{s}$.

Second, we checked how many different best frequencies were represented, if ITD peaks occurring at 0 (Fig. $7 A$ ), at $-100 \mu \mathrm{s}$
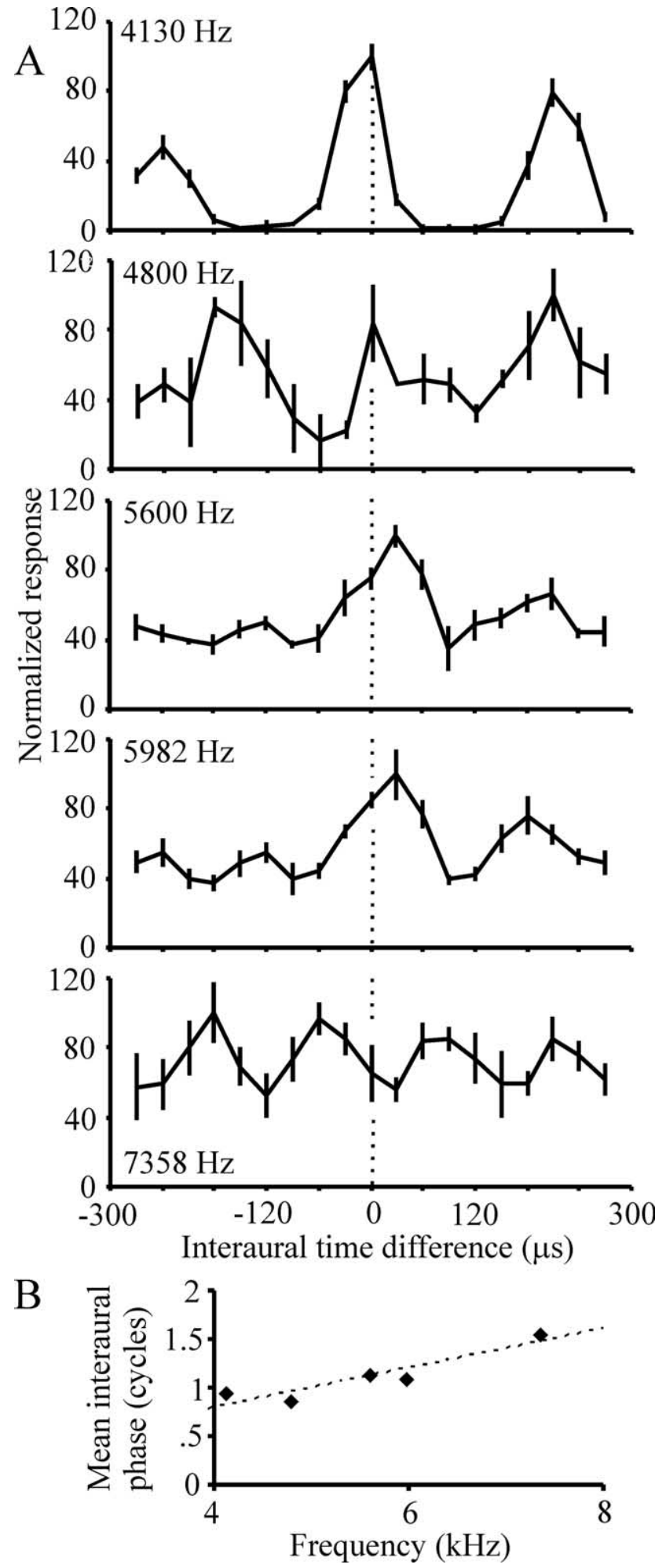

Figure 4. ITD tuning in a dorsoventral penetration through ICCls. $\boldsymbol{A}$, Tuning curves from the ICCls were arranged similarly as in ICCC, but the array-specific ITD had the opposite sign $(\boldsymbol{B})$. The recordings came from the left brain side. The correlation coefficient was 0.93 , the array-specific ITD amounted to $201 \mu \mathrm{s}$, and the characteristic phase was 0.01 . Note that the $x$-axis is truncated at $4 \mathrm{kHz}$.

(Fig. $7 B$ ), at $-200 \mu \mathrm{s}$ (Fig. $7 C$ ), or at $-400 \mu$ s (Fig. $7 D$ ) were selected. We first noted that, in all four cases (Fig. $7 A-D)$, the total low-frequency range $(333-2500 \mathrm{~Hz})$ was represented. Consequently, the MIPD varied considerably and with it the relative 

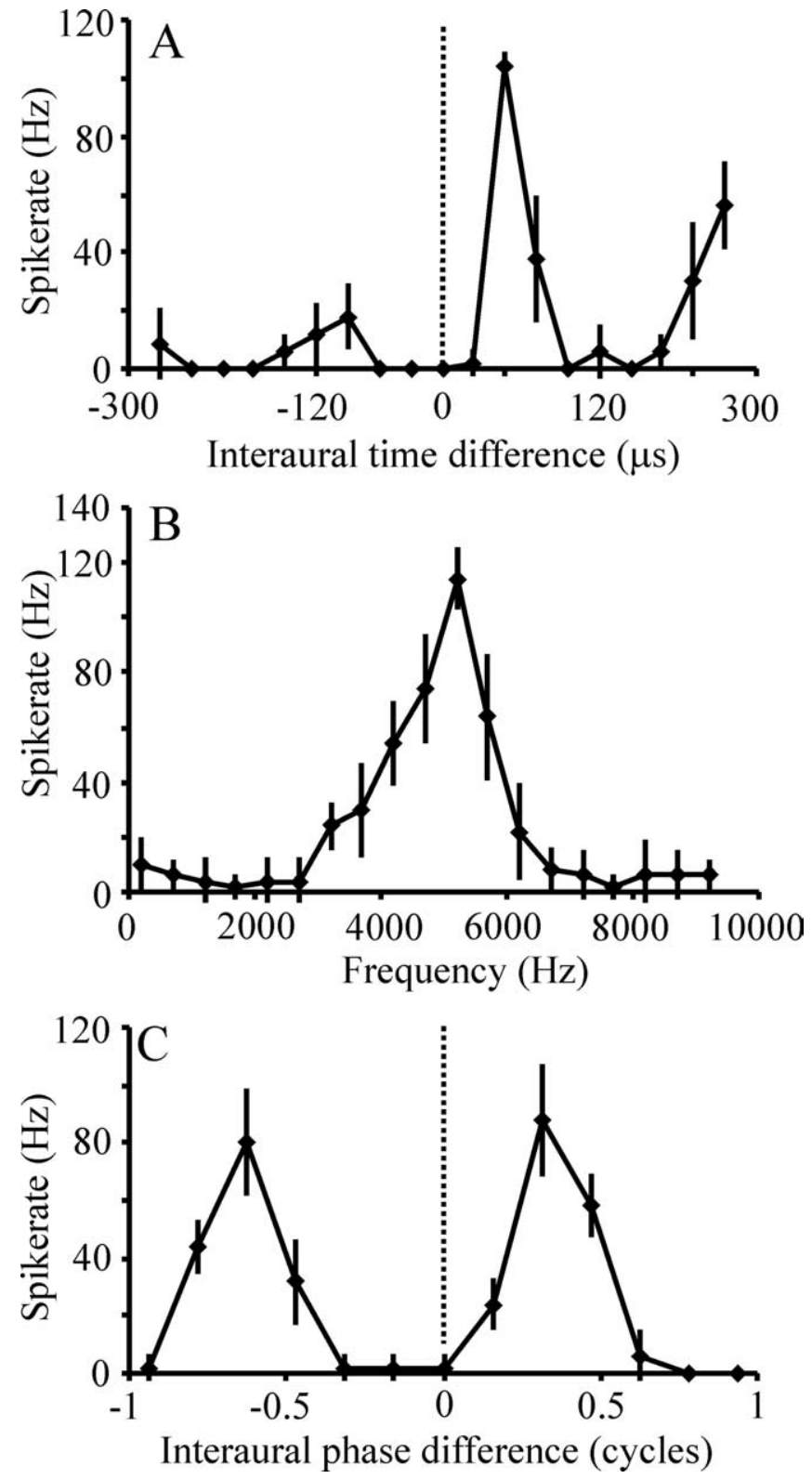

Figure 5. Responses of a high-frequency ICX neuron. $\boldsymbol{A}-\boldsymbol{C}$, Same records as in Figure 1. Note the side-peak suppression $(\boldsymbol{A})$, the relatively broad frequency tuning $(\boldsymbol{B})$, and the cyclic response to tone stimulation (C). Each stimulus was repeated five times. Data are from a single unit.

response level at 0 ITD. For example, in the case of the peak occurring at an ITD of $-100 \mu$ s, the 0 ITD line was crossed at a response level of $80 \%$ relative to the response maximum ( 88 spikes/s) and an interaural phase difference of -0.15 cycles in the unit with a $\mathrm{BF}$ of $714 \mathrm{~Hz}$ (Fig. $7 \mathrm{~B}$, dashed line), whereas in the unit with a $\mathrm{BF}$ of $2500 \mathrm{~Hz}$, the response level at 0 ITD was $7 \%$ and the interaural phase difference was -0.25 (Fig. $7 B$, dot-dot-dashed line; response maximum, 54 spikes/s). Similar calculations may be made for the responses displayed in Figure 7, $C$ and $D$, with the result that many different relative response levels and many different interaural phase differences were represented at 0 ITD.

If all 637 data points obtained in ICCc with noise stimulation were displayed in one plot, a clustering of ITD peaks could not be seen (Fig. 8A). Not only did ITD peaks not cluster, but also the distribution of MIPD in the low-frequency region covered a wide range (Fig. $8 B$ ). When histograms of the MIPD from different
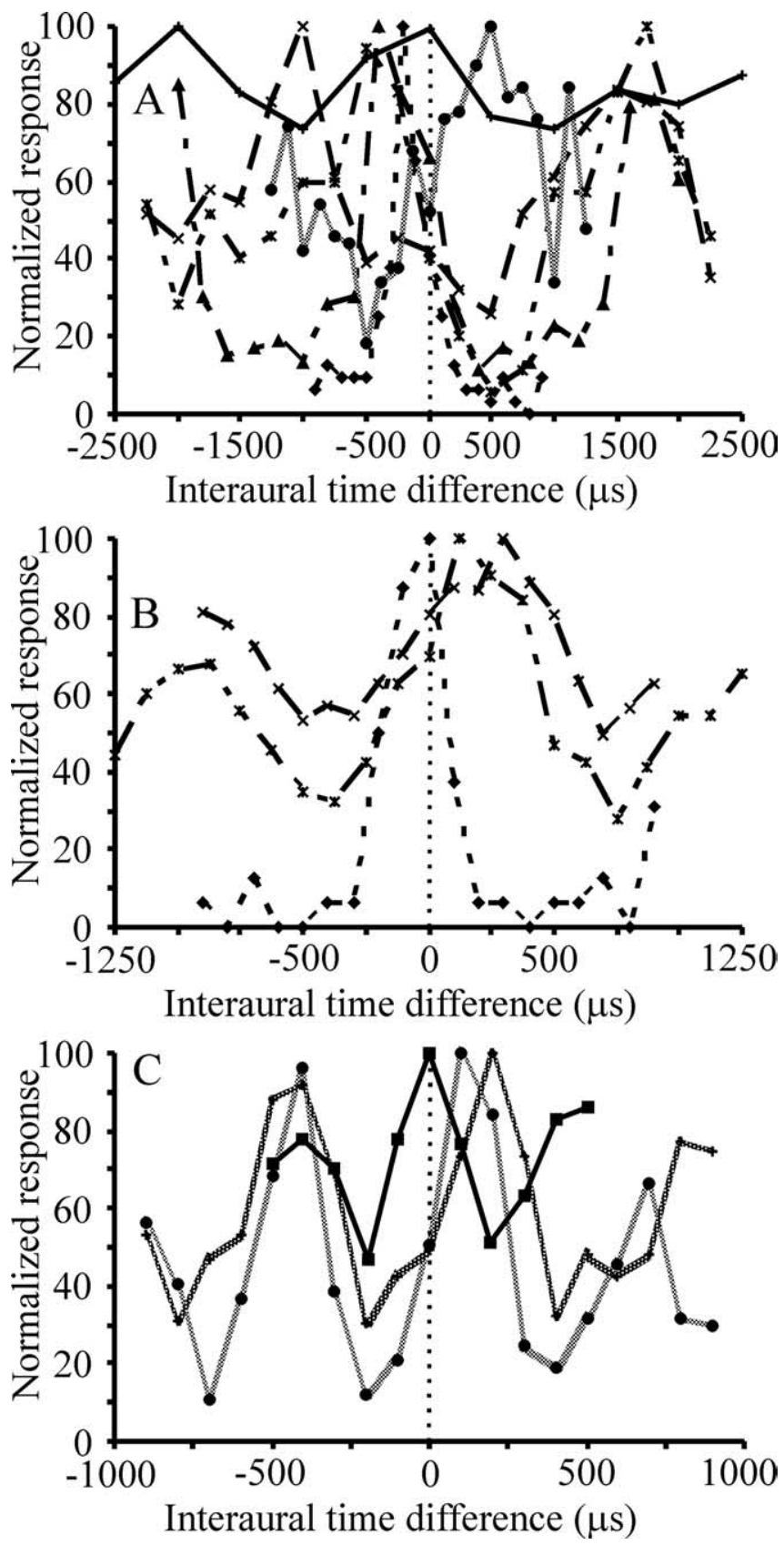

Figure 6. Distribution of ITD peaks at different frequencies. Neurons tuned to $400 \mathrm{~Hz}(\boldsymbol{A}), 800$ $\mathrm{Hz}(\boldsymbol{B})$, and $1667 \mathrm{~Hz}(\boldsymbol{C})$ were selected. The neurons in $\boldsymbol{A}$ came from both brain sides, whereas the neurons in $\boldsymbol{B}$ and $\boldsymbol{C}$ came from the right brain side only. In every frequency band, peaks were found not only scattered around one ITD, but covered a large range of ITDs. All data were obtained with noise stimulation from units located in ICCC.

frequency regions were compiled, this situation became even clearer (Fig. 8C). However, 0 MIPD was overrepresented in the low-frequency range. In the high-frequency range (439 data points), the number of cases in the five columns scattered between 15 and $24 \%$, close to the expected 20\% for each class, if an equal distribution were assumed.

The advantage of noise stimulation is that it closely represents the natural situation. However, the use of noise leads to some imprecision in determining the frequency to be used in a plot like the one shown in Figure $8 \mathrm{~A}$. Tonal stimuli, in contrast, are unnatural stimuli but have the advantage that the frequency is exactly known. Therefore, similar plots as for the noise data (Fig. 
$8 A$ ) were compiled with the tone data (Fig. $9 A)$. For these plots, MIPDs were obtained from circular statistics and limited to \pm 0.5 cycles. The corresponding ITDs were then calculated by dividing the MIPD by the stimulation frequency. Consequently, ITDs were restricted to the $\pi$ limit. These analyses yielded similar results to the noise data (compare Figs. $8 A, C ; 9 A, B$ ): Peaks of ITDs were scattered over the entire possible ITD range (Fig. 9A). The distribution of the mean interaural phase differences was similar to the noise data, but in the low-frequency range 0 MIPD did not occur as often as in the noise data. In the high-frequency range, the occurrence of MIPDs (243 data points) in the five classes varied between 16 and 25\% (Fig. 9B).

\section{Distribution of ITD peaks and best}

frequencies in ICCls and ICX

Analyses similar to those for ICCc responses were performed for the noise responses in ICCls (Fig. 10A,B) and ICX (Fig. 10C,D). In ICCls, best frequencies covering the whole frequency range were observed (Fig. 10A), but a smaller portion of the neurons than in ICCc had best frequencies $<2500 \mathrm{~Hz}$ (198 of 637 or $31.1 \%$ in ICCc vs 76 of 438 or $17.4 \%$ in ICCls). The lowest BF in our sample from ICCls was $500 \mathrm{~Hz}$. There are only 10 data points for the frequency range $<800 \mathrm{~Hz}$, indicating again that the very low frequencies were rare in the ICCls. The distribution of peaks for frequencies $<2500 \mathrm{~Hz}$ in ICCls (76 data points) was similar as in ICCc, with a broad distribution from 0 to 0.5 cycles. The missing low frequencies also caused the ITD range represented to shrink from 1500 to $<750 \mu$ s (compare Figs. $8 A, 10 A$ ). This reflects the fact that the peaks at the very extreme ITDs (less than -1000 or $>1000 \mu \mathrm{s}$ ) were all observed in units having a $\mathrm{BF}<450 \mathrm{~Hz}$ (Fig. $8 A)$. Again, in the high-frequency range, the percentages in the five classes observed were close to the predicted values when an equal distribution was assumed (Fig. 10C) (20\% predicted; $16-$ $23 \%$ observed; total number of data points, 362 ).

In ICX, the trend toward a representation of higher frequencies was even more dramatic than in ICCls (compare Fig. $10 \mathrm{C}$ with Figs. 10A, 8A). Only 5 of 588 units $(<1 \%)$ had a BF $<2500$ $\mathrm{Hz}$ in the sample from ICX. This again led to shrinkage of the range of ITDs represented in ICX compared with ICCls. A peak at an ITD of $215 \mu$ s represented the largest absolute value of the ITD observed in ICX (Fig. 10 C). The distribution of the peaks in the high-frequency range was broad. However, 0 MIPD was overrepresented (Fig. 10D).

\section{Response peaks outside the $\pi$ limit}

In Figures $8 A$ and 10, $A$ and $C$, the $\pi$ limit is indicated by the solid lines. These borders reflect processing within one period, measured at the BF of a unit. The data demonstrated that responses outside the $\pi$ limit occurred in all three subnuclei. The lowest frequency at which a peak outside the $\pi$ limit was observed was in ICCc at a BF of $930 \mathrm{~Hz}$. Although the ITD range represented decreased with increasing $\mathrm{BF}$ in all three subnuclei, even at high BFs peaks outside the $\pi$ limit were observed. The highest BF for which this was the case was $8600 \mathrm{~Hz}$.
In the cases in which a peak occurred outside the $\pi$ limit, a second peak within the $\pi$ limit was present. Therefore, the way of determining why the peak outside the $\pi$ limit was chosen as relevant needs explanation. We based our decision on several criteria. For the cells in ICCc and ICCls, we used the arrayspecific ITDs as the primary criteria for defining the peaks, as stated previously. If peaks in the scatter diagrams (Figs. 2-4) from which the array-specific ITD was calculated occurred for some units beyond +0.5 or -0.5 cycles (Fig. 2 , arrows), then this meant that the peak outside the $\pi$ limit was the relevant peak and not the peak within the $\pi$ limit.

For ICX, an additional criterion was available. In ICX, there is a main, behaviorally relevant peak, and one or more side peaks. The response at the side peaks is suppressed (Fig. 5A). Thus, we used the main peak as the relevant peak in ICX units. In the responses from the penetration shown in Figure 11, we recorded noise tuning curves at three locations, and a tone tuning curve at location 1 . The responses to the tonal stimulation were lower than the response to the noise stimulation, typical for ICX. After the recordings, a large lesion was made close to location 1 . This lesion caused a behavioral deficit for location more peripheral than $45^{\circ}$ of azimuth (Wagner, 1993). The center frequency at location 1 was $5555 \mathrm{~Hz}$, and the frequency range represented at location 1 was from 4000 to $8000 \mathrm{~Hz}$. The gray area indicates the $\pi$ limit for the center frequency. The peak in the tone ITD curve occurred at $90 \mu$ s or exactly at the $\pi$ limit. The peak in the noise ITD curve at location 1 lay between 90 and $120 \mu$ s. At locations 2 and 3 , the peak in the noise ITD curve was found at $120 \mu$ s. Note side-peak suppression in the noise ITD curves with side peaks occurring between -60 to $-120 \mu$ s (height between 18 and $52 \%$ of height of main peak), which is close to the secondary peak in the tone ITD curve. Thus, for the higher frequencies $(>5 \mathrm{kHz})$ considered to be behaviorally relevant (Konishi, 1973; Knudsen et al., 1979), strong responses outside the $\pi$ limit occurred. 

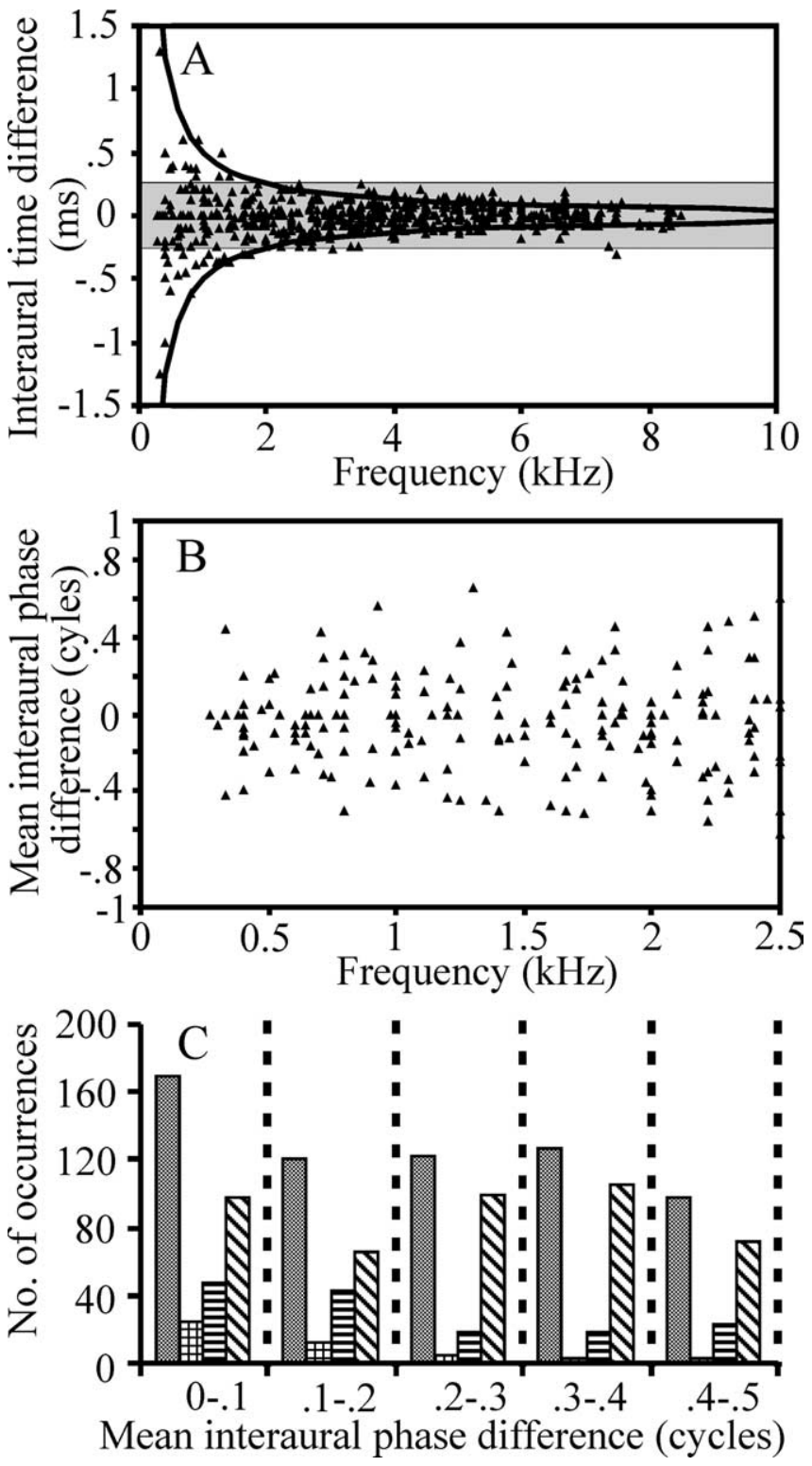

Figure 8. Distribution of ITD peaks as a function of best frequency in noise responses in ICCC $A$, Peaks of ITD (637 units) were distributed over a wide range. The shaded area indicates the physiological ITD range $(-250$ to $+250 \mu \mathrm{s})$. At the lowest frequencies, peaks occurred outside this range. The two lines indicate the ITD at \pm 0.5 cycles, also known as the $\pi$ limit. A considerable number of peaks measured at the best frequency with noise stimuli occurred outside the $\pi$ limit. $\boldsymbol{B}$, Distribution of MIPDs in the low-frequency range. $\boldsymbol{C}$, The distribution of noise peaks in different frequency bands (dotted: all, 637 data points; cross-hatched: $\mathrm{BF}<800 \mathrm{~Hz}$, 48 data points; horizontal line: $800 \mathrm{~Hz} \leq \mathrm{BF}<2500 \mathrm{~Hz}, 150$ data points; back slashes, BF $>2500 \mathrm{~Hz}$, 432 data points). Note that, for $C$, MIPDs were calculated, and the range of MIPDs was restricted to -0.5 to 0.5 cycles by phase wrapping. Then, left-right symmetry was assumed, and the absolute value of the mean interaural phase was plotted.

\section{Discussion}

The representation of interaural time difference was studied in the inferior colliculus of the barn owl. Response peaks were widely scattered. The representation of ITD in high-frequency neurons, but not in low-frequency neurons followed predictions of optimal coding. In all three subnuclei of the IC, responses lying outside the $\pi$ limit were observed. These findings will be discussed with respect to models of the computation and representation of ITD in both mammals and birds and with respect to the possible role of behavioral constraints on optimal coding.
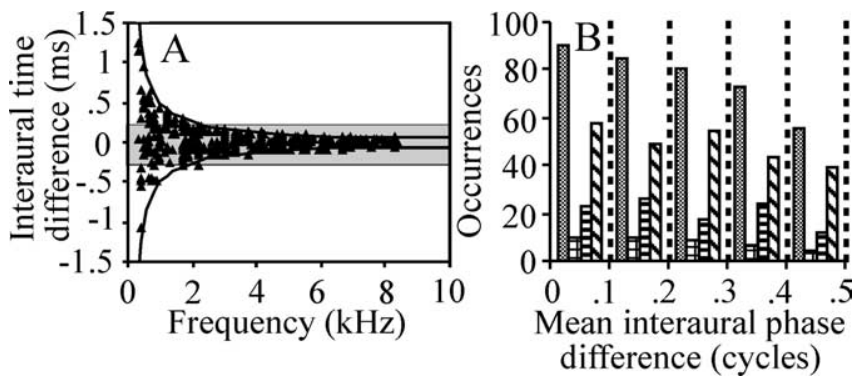

Figure 9. Distribution of ITD peaks as a function of best frequency in tone responses in ICCC. A, Peaks of ITD from ITD curves (384 units). The shaded area indicates an ITD range from -250 to $+250 \mu \mathrm{s}$. The two lines indicate the ITD at \pm 0.5 cycles, also known as the $\pi$ limit. Note that, for this plot, first MIPDs in a range covering \pm 0.5 cycles were obtained. The ITDs were then calculated from the MIPDs by dividing the MIPD by the stimulation frequency. This recalculation was done to obtain a more precise measure of the best ITD of a unit than reading the peak value from the ITD curve. $\boldsymbol{B}$, The distribution of tone peaks in different frequency bands (dotted: all, 384 data points; cross-hatched: $\mathrm{BF}<800 \mathrm{~Hz}, 39$ data points; horizontal line: $800 \mathrm{~Hz} \leq \mathrm{BF}<$ $2500 \mathrm{~Hz}, 102$ data points; back slashes, BF $>2500 \mathrm{~Hz}, 243$ data points). The data in $\boldsymbol{B}$ were calculated as described in Figure $8 \mathrm{C}$.
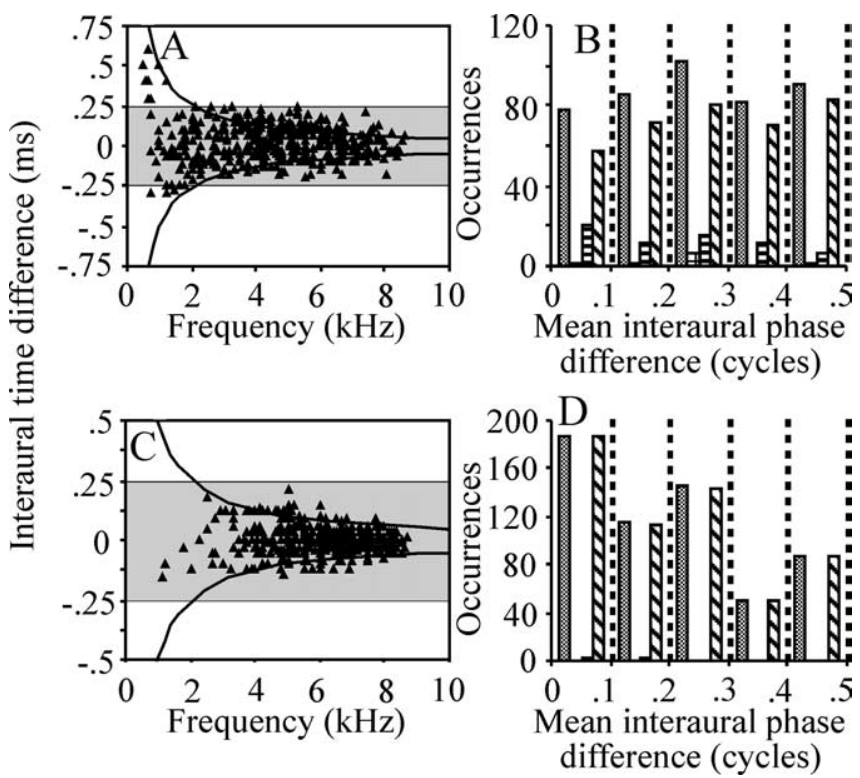

Figure 10. Distribution of peaks in the ICCIs and ICX. $A, B$, Distribution of peaks in ICCIS ( 438 units). $C, D$, Distribution of peaks in ICX (588 units). Data were obtained as for Figure $8, A$ and $C$, from noise ITD curves. Note that, in ICX, frequencies $<2.5 \mathrm{kHz}$ were very rare. Note also that, in the low-frequency range, some peaks measured at the best frequency occurred outside the physiological ITD range. In the high-frequency range, peaks occurred outside the $\pi$ limit. The data in $\boldsymbol{B}$ and $\boldsymbol{D}$ were calculated as described in Figure $8 C$.

\section{Representation of ITD in the barn owl}

The data presented here have mainly been collected for other projects. This raises the question whether these data contain sampling biases. We think this possibility unlikely for several reasons. First, the integration of all the available data into a single large data base should reduce variability because the data were collected for independent projects. Second, the results presented here are consistent in several respects with conclusions drawn from previous studies. For example, it has been reported previously that ITD is mapped in the IC (Knudsen and Konishi, 1978a; Wagner et al., 1987, 2002; Takahashi et al., 1989; Fujita and Konishi, 1991) as well as in nucleus laminaris (Sullivan and Konishi, 1986; Carr and Konishi, 1990) that provides input to the IC. All 


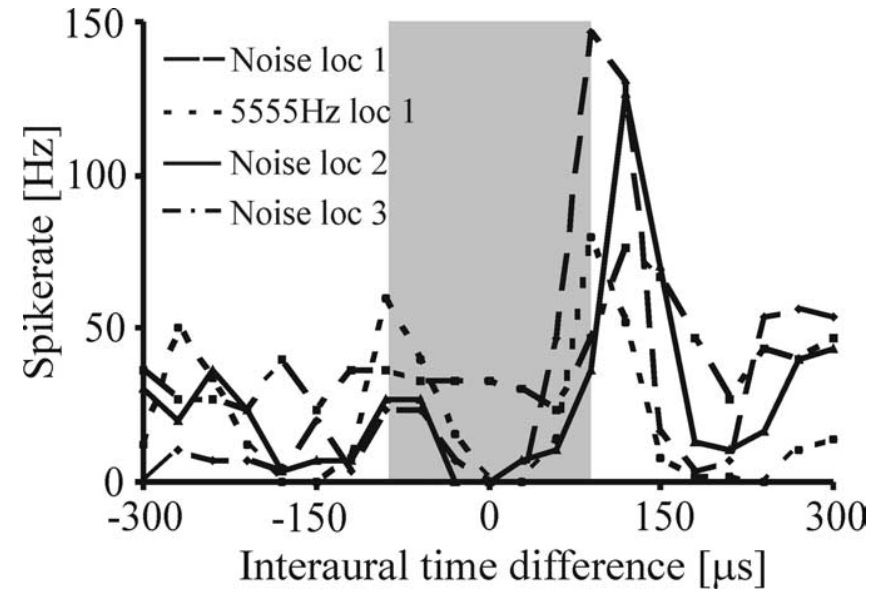

Figure 11. Responses outside the $\pi$ limit. Responses from a penetration through the ICX, as verified by a lesion, are shown. Responses were obtained at the three locations (loc 1, loc 2, loc 3). At location 1, a noise and a tone curve were measured, whereas at locations 2 and 3 only data for noise-tuning curves were collected. The center frequency at location 1 was $5555 \mathrm{~Hz}$, corresponding to a period of $180 \mu \mathrm{s}$. The gray area indicates the range from -90 to $90 \mu \mathrm{s}$, corresponding to the $\pi$ limit at the center frequency at location 1 . Note that strong responses occurred outside the $\pi$ limit.

three subnuclei of the IC showed a continuous distribution of MIPD phase throughout the entire frequency range, consistent with a map-like representation of ITD. The overrepresentation of 0 MIPD reported here is also in line with previous findings (Knudsen and Konishi, 1978a).

The same distribution of MIPDs was found in the lowfrequency range. Data from only a limited number of cells with BFs $<2500 \mathrm{~Hz}$ range were previously available (Wagner et al., 2002) (see critique by Harper and McAlpine, 2004). For this report, the number of units in the database was increased by a factor of 4 . Within this sample, no indications of preferred MIPDs other than around 0 MIPD were observed. No sign of a break in the representation could be seen in the frequency range (Figs. 8-10). This observation is important because the model of Harper and McAlpine (2004) predicted such a break should occur between 2000 and $3000 \mathrm{~Hz}$. This model further predicted concentrations of best MIPDs around preferred values for best frequencies $<2000-3000 \mathrm{~Hz}$. For example, at $400 \mathrm{~Hz}$, a preferred phase around 0.16 MIPD was predicted. Eight data points were obtained for this frequency, of which none lies between an MIPD of 0.1 and 0.3 MIPD. For the frequency range between $\sim 400$ and $800 \mathrm{~Hz}$, we also observed a wide distribution covering the whole MIPD range and not a concentration of data points from 0 to 0.2 MIPD as the model suggested. Last but not least, in the range between 1000 and $2000 \mathrm{~Hz}$, the two peaks predicted by the model were not observed.

\section{Relationship to models of the representation of ITD}

Several models of the representation of ITD have been proposed (Jeffress, 1948; Colburn et al., 1990; Fitzpatrick et al., 1997; McAlpine et al., 2001; Grau-Serrat et al., 2003; Harper and McAlpine, 2004). Of specific interest here is a comparison between the slope-code model (McAlpine et al., 2001; Harper and McAlpine, 2004; McAlpine, 2005) and the place-code model (Jeffress, 1948). Our data do not contradict the place-code model, but do not support some predictions of the slope-code model. In the previous section, we discussed the prediction of a scattering of response peaks around preferred MIPDs in the low-frequency range by the slope-code model. We did not observe this. In contrast, the place-code model predicts our observations of broad MIPD distributions. Moreover, if a slope code were realized, the responses we observed outside the $\pi$ limit were not expected. However, the slope-code model predicts peaks outside the physiological range (see also Carr and Koppl, 2004), as we observed. We argue here that an observation of peaks outside the physiological ITD range is not sufficient for the conclusion that a slope code is implemented. Specifically, the place-code model can handle such peaks, although they might not be expected to occur, because they do not have an associated location in space. Relevant to this discussion is the observation that owls are able to discriminate artificial ITDs five times larger that the physiological ITD range (Saberi et al., 2002). It is possible that the large ITDs are still useful for discriminating left from right. However, neurons with peaks outside the physiological ITD range may also convey information other than sound localization (Fitzpatrick et al., 2000).

Our conclusion does not mean that the owl does not have access to the information contained in the slopes of the ITD curves. Indeed, Bala et al. (2003) demonstrated that barn owls assess this information to discriminate changes in the location of sound sources as small as $3^{\circ}$ in azimuth.

\section{Response outside the $\pi$ limit}

Some of the response maxima occurred outside the $\pi$ limit. Peak positions outside the $\pi$ limit are expected in a place-code model for frequencies for which the physiological ITD range exceeds the $\pi$ limit. This would be the case in the owl for frequencies above $\sim 2-3 \mathrm{kHz}$ (von Campenhausen and Wagner, 2006). Many reports have found that the owl preferentially uses frequencies between 5 and $9 \mathrm{kHz}$ for sound localization (Konishi, 1973; Saberi et al., 1999; Poganiatz et al. 2001; Bala et al., 2003). That the peaks outside the $\pi$ limit are relevant is concluded from the analysis of ITD arrays as shown in Figures 2-4 (see also Wagner et al., 1987; Takahashi et al., 1989). Sometimes the behavioral relevance of the responses was known (Wagner, 1993).

A word of caution may be appropriate here, nevertheless. It is not clear what the relevant frequency is in broadband neurons like those in ICX. We calculated the MIPD with respect to the center frequency of these neurons. It might be argued that the owl would only use the low-frequency information present in these neurons. If so, the responses as shown in Figure 11 would not lie outside the $\pi$ limit. We consider this possibility remote, because the frequency range between 5 and $9 \mathrm{kHz}$ underlies the precise sound localization in the barn owl. The situation may, however, be even more complex: Thompson et al. (2006) showed for humans that, as the ITD of a sound increased, the peak of activity switched brain sides, although the perceived location remained on the same side. Thus, the amplitude of midbrain activity was not related to perception in humans.

\section{Considerations on optimal coding}

If we accept that optimal coding is a good strategy for biological systems, we are forced to conclude that the model by Harper and McAlpine (2004) did not take into account all necessary constraints. One of these neglected constraints is behavior. As mentioned previously, barn owls use mainly high-frequency sounds for locating potential prey (Konishi, 1973). A representation consistent with optimal coding is present in this frequency range. The role of low-frequency information in sound localization is unclear (Payne, 1971; Bremen et al., 2005). Experience-dependent plasticity shapes the representation of ITD (Knudsen, 1999; Seidl and Grothe, 2005). We suggest that in the low-frequency range of 
the barn owl the crude representation formed by genetic information persists in adulthood, because there is not enough evolutionary pressure to establish the optimal coding principle. This conclusion suggests that the predictions made in the model (Harper and McAlpine, 2004) for the other species should undergo additional scrutiny that takes into account ecological constraints and behavior. In this context, it is notable that gerbils may have evolved low-frequency hearing in response to high predation pressure (Lay, 1974), consistent with the implementation of an optimal coding algorithm in the low-frequency range. However, the predatory behavior of the cat, the third animal mentioned in Harper and McAlpine (2004), is more complex, including both visual and auditory cues. Thus, a solution for the organization of the sound localization system may be difficult to predict. This may in part explain the continued discussion on the representation of ITD in the cat (Yin and Kuwada, 1984; Bradshaw, 1992; Hancock and Delgutte, 2004). Similar considerations may apply for other species.

\section{References}

Bala AD, Spitzer MW, Takahashi TT (2003) Prediction of auditory spatial acuity from neural images on the owl's auditory space map. Nature 424:771-774.

Batra R, Yin TC (2004) Cross correlation by neurons of the medial superior olive: a reexamination. J Assoc Res Otolaryngol 5:238-252.

Bradshaw JWS (1992) The behavior of the domestic cat. Melksham, UK: Redwood.

Brand A, Behrend O, Marquardt T, McAlpine D, Grothe B (2002) Precise inhibition is essential for microsecond interaural time difference coding. Nature 417:543-547.

Bremen P, Singheiser M, Plachta DTT, van der Willigen RF, Wagner $\mathrm{H}$ (2005) Barn owls do not depend on high frequency auditory signals to approach a target. Proceedings of the 6th Meeting of the German Neuroscience Society/30th Goettingen Neurobiology Conference. 2005:464.

Bremen P, Poganiatz I, von Campenhausen H, Wagner H (2006) Sensitivity to interaural time difference and representation of azimuth in central nucleus of inferior colliculus in the barn owl. J Comp Physiol A Neuroethol Sens Neural Behav Physiol 193:99-112.

Carr CE, Konishi M (1990) A circuit for detection of interaural time differences in the brainstem of the barn owl. J Neurosci 10:3227-3246.

Carr CE, Koppl C (2004) Coding interaural time differences at low best frequencies in the barn owl. J Physiol (Paris) 98:99-112.

Colburn HS, Han Y, Culotta CP (1990) Coincidence model of MSO responses. Hear Res 49:335-346.

Fitzpatrick DC, Batra R, Stanford TR, Kuwada S (1997) A neuronal population code for sound localization. Nature 388:871-874.

Fitzpatrick DC, Kuwada S, Batra R (2000) Neural sensitivity to interaural time differences: beyond the Jeffress model. J Neurosci 20:1605-1615.

Fujita I, Konishi M (1991) The role of GABAergic inhibition in processing of interaural time difference in the owls auditory system. J Neurosci 11:722-739.

Goldberg JM, Brown PB (1969) Response of binaural neurons of dog superior olivary complex to dichotic tonal stimuli: some physiological mechanisms of sound localizations. J Neurophysiol 32:613-636.

Grau-Serrat V, Carr CE, Simon JZ (2003) Modelling coincidence detection in nucleus laminaris. Biol Cybern 89:388-396.

Hancock KE, Delgutte B (2004) A physiologically based model of interaural time difference discrimination. J Neurosci 24:7110-7117.

Harper NS, McAlpine D (2004) Optimal neural population coding of an auditory spatial cue. Nature 430:682-686.

Jeffress LA (1948) A place theory of sound localization. J Comp Physiol Psychol 41:35-39.

Joris PX, Smith PH, Yin CT (1998) Coincidence detection in the auditory system: 50 years after Jeffress. Neuron 21:1235-1238.

Kautz D, Wagner H (1998) GABAergic inhibition influences auditory motion-direction sensitivity in barn owls. J Neurophysiol 80:172-185.

Keller CH, Takahashi TT (2005) Localization and identification of concurrent sounds in the owl's auditory space map. J Neurosci 25:10446-10461.

Keller CH, Hartung K, Takahashi TT (1998) Head-related transfer func- tions of the barn owl: measurement and neural responses. Hear Res 118:13-34.

Knudsen EI (1984) Synthesis of a neural map of auditory space in the owl. In: Dynamic aspects of neocortical function (Edelman GM, Gall EW, Cowen MW, eds), pp 375-396. New York: Neurosciences Research Foundation, Inc.

Knudsen EI (1999) Mechanism of experience-dependent plasticity in the auditory localization pathway of the barn owl. J Comp Physiol A 185:305-321.

Knudsen EI, Konishi M (1978a) A neural map of auditory space in the owl. Science 200:795-797.

Knudsen EI, Konishi M (1978b) Space and frequency are represented separately in auditory midbrain of the owl. J Neurophysiol 41:870-884.

Knudsen EI, Basdel GG, Konishi M (1979) Sound localization by the barn owl (Tyto alba) measured with the search coil technique. J Comp Physiol A 133:1-11.

Konishi M (1973) How the owl tracks its prey. Am Scientist 61:414-424.

Lay DM (1974) Differential predation on gerbils (Meriones) by the little owl, Athene brahma. J Mammalogy 55:608-614.

McAlpine D (2005) Creating a sense of auditory space. J Physiol (Lond) 566:21-28.

McAlpine D, Jiang D, Palmer AR (2001) A neural code for low-frequency sound localization in mammals. Nat Neurosci 4:396-401.

Moiseff A, Konishi M (1981) Neuronal and behavioral sensitivity to binaural time differences in the owl. J Neurosci 1:40-48.

Moiseff A, Konishi M (1983) Binaural characteristics of units in the owl's brainstem auditory pathway: precursors of restricted spatial receptive fields. J Neurosci 3:2553-2562.

Payne R (1971) Acoustic localization of prey by barn owls (Tyto alba). J Exp Biol 54:535-573.

Poganiatz I, Nelken I, Wagner H (2001) Sound-localization experiments with barn owls in virtual space: influence of interaural time difference on head-turning behavior. J Assoc Res Otolaryngol 2:1-21.

Rose JE, Gross NG, Geisler CD, Hind JE (1966) Some neural mechanisms in the inferior colliculus of the cat which may be relevant to the localization of a sound source. J Neurophysiol 29:288-314.

Saberi K, Takahashi Y, Farahbod H, Konishi M (1999) Neural bases of an auditory illusion and its elimination in owls. Nat Neurosci 2:656-659.

Saberi K, Takahashi Y, Egnor R, Farahbod H, Mazer J, Konishi M (2002) Detection of large interaural delays and its implication for models of binaural interaction. J Assoc Res Otolaryngol 3:80-88.

Seidl AH, Grothe B (2005) Development of sound localization mechanisms in the mongolian gerbil is shaped by early acoustic experience. J Neurophysiol 94:1028-1036.

Siveke I, Pecka M, Seidle AH, Baudoux S, Grothe B (2006) Binaural response properties of low-frequency neurons in the gerbil dorsal nucleus of the lateral lemniscus. J Neurophysiol 96:1425-1440.

Stecker HC, Harrington IA, Middlebrokks JC (2005) Location coding by opponent neural populations in the auditory cortex. PLoS Biol 3:520-528.

Sullivan WE, Konishi M (1986) Neural map of interaural phase difference in the owl's brainstem. Proc Natl Acad Sci USA 83:8400-8404.

Takahashi T, Konishi M (1986) Selectivity for interaural time difference in the owl's midbrain. J Neurosci 6:3413-3422.

Takahashi TT, Wagner H, Konishi M (1989) Role of commissural projections in the representation of bilateral auditory space in the barn owl's inferior colliculus. J Comp Neurol 281:545-554.

Thompson SK, von Kriegstein K, Deane-Pratt A, Marquardt T, Deichmann R, Griffiths TD, McAlpine D (2006) Representation of interaural time delay in the human auditory midbrain. Nat Neurosci 9:1096-1098.

von Campenhausen M, Wagner H (2006) Influence of the facial ruff on the sound-receiving characteristics of the barn owl's ears. J Comp Physiol A 192:1073-1082.

Wagner H (1990) Receptive fields of neurons in the owl's auditory brainstem change dynamically. Eur J Neurosci 2:949-959.

Wagner H (1993) Sound-localization deficits induced by lesions in the barn owl's space map. J Neurosci 13:371-386.

Wagner H, Takahashi T (1990) Neurons in the midbrain of the barn owl are sensitive to the direction of apparent acoustic motion. Naturwissenschaften 77:439-442.

Wagner H, Takahashi TT (1992) Influence of temporal cues on acoustic 
motion-direction sensitivity of auditory neurons in the owl. J Neurophysiol 68:2063-2076.

Wagner H, von Campenhausen M (2002) Distribution of auditory motiondirection sensitive neurons in the barn owl's midbrain. J Comp Physiol A 188:705-713.

Wagner H, Takahashi T, Konishi M (1987) Representation of interaural time difference in the central nucleus of the barn owl's inferior colliculus. J Neurosci 7:3105-3116.

Wagner H, Trinath T, Kautz D (1994) Influence of stimulus level on acoustic motion-direction sensitivity in barn owl midbrain neurons. J Neurophysiol 71:1907-1916.

Wagner H, Mazer JA, von Campenhausen M (2002) Response properties of neurons in the core of the central nucleus of the inferior colliculus of the barn owl. Eur J Neurosci 15:1343-1352.

Wagner H, Güntürkün O, Nieder B (2003) Anatomical markers for the subdivisions of the barn owl's inferior-collicular complex and adjacent periand subventricular structures. J Comp Neurol 465:145-159.

Yin TC, Kuwada S (1984) Neuronal mechanisms of binaural interactions. In: Dynamic aspects of neocortical function (Edelman GM, Gall WE, Cowan WM, eds), pp 263-313. New York: Wiley.

Zhou Y, Carney LH, Colburn HS (2005) A model for interaural time difference sensitivity in the medial superior olive: interaction of excitatory and inhibitory synaptic inputs, channel dynamics, and cellular morphology. J Neurosci 25:3046-3058. 